\title{
Frequency of bacterial species associated with nosocomial infection among health care personnel in Nigeria
}

\begin{abstract}
Background and objective: Nosocomial infections are a serious public health issue today and its studies cannot be overemphasized. An open label 12-weeks descriptive crosssectional survey was conducted in a large number of hospital Personnel to determine the frequency of bacterial species associated with nosocomial infections among healthcare personnel in the north central geopolitical zone of Nigeria.
\end{abstract}

Materials and method: The subjects in this study were forty males, forty females and nineteen of the hospital fomites. The moistened swab sticks were separately robbed on the hands of forty that washed with soap and water and forty that washed with disinfectants and nineteen of the hospital fomites. The samples were inoculated in different laboratory media and incubated at $37^{\circ} \mathrm{C}$ for 48 hours. Morphological, cultural and biochemical characteristics were noted appropriately. Means and corresponding standard deviations were calculated for continuous data while proportions, along with the $95 \%$ confidence intervals, were calculated for categorical data.

Results: This study shows that microorganisms occupy the hands of social insurance work force and gear and materials being used in clinics. The major types of organisms isolated from the hands of the health care personnel and the hospital fomites in this study were Staphylococcus aureus, Eschenchia coli, Klebsiellapneumoniae and Pseudomonas spp. The most frequently isolated was Staphylococcus aureus.

Conclusion: To ensure proper hygiene, medical personnel should consider staff decontamination following a period of nursing of patients especially if they are to attend to unaffected patients shortly

Keywords: hospital acquired infections, staff decontamination, hand washing
Volume 5 Issue 6 - 2017

\author{
Itodo Sunday Ewaoche,' Iquo Bassey Otu \\ Bassey,' Esther Uchenna Umeh² \\ 'Department of Medical Laboratory Science, University of \\ Calabar, Nigeria \\ ${ }^{2}$ Department of Biological Sciences, University of Agriculture \\ Makurdi, Nigeria
}

\author{
Correspondence: Itodo Sunday E, Department of Medical \\ Laboratory Science, Faculty of Allied Medical Sciences, \\ University of Calabar, Calabar, Nigeria, Tel 234802986953 I, \\ Email ewieise@gmail.com
}

Received: September II, 2017| Published: October 30, 2017
Abbreviations: HAI, health care-associated infections; UTIs, urinary tract infections

\section{Introduction}

Nosocomial infections are a serious public health issue today. They are a major cause of morbidity and mortality. Their pathologies are responsible for considerable increase in health care expenses despite the widely recognized efforts of public health authorities to keep them under control. ${ }^{1}$ Almost two million nosocomial infections are detected every year in the United States, 80,000 of which result in the patient's premature death. ${ }^{2}$ Their total annual costs for five major health careassociated infections (HAIs) were $\$ 9.8$ billion, with surgical site infections contributing the most to overall costs. ${ }^{3}$

Five million hospital acquired infections are detected in Europe every year, 135,000 of which end in death. Almost 1.4 million people worldwide suffer from a nosocomial infection every day. ${ }^{4}$ Microorganisms are ubiquitous occurring nearly everywhere in nature. They occur most abundantly in the presence of nutrients, moisture and temperature suitable for their growth and multiplication. ${ }^{5}$ Since the conditions that favour the growth and multiplication of most microorganisms are also favourable to man, it is inevitable that we live among thousands of microorganisms. They are found in the air we breathe, the food we eat and on our body surfaces and other close environments. ${ }^{6}$ The presence of microorganisms in a location has various effects that could either be beneficial or harmful and human beings have various ways of resisting invasion by potentially harmful ones.

In the hospital environment, the presences of microorganisms are usually not beneficial. They cause infections that subsequently lead to disease in susceptible patients. Microorganisms are present in the hospital environment including surfaces of sinks, taps, bedpans, beds, floors, bedspreads, stethoscopes, chairs, cupboards, theatre rooms, on surgical instrument ${ }^{7}$ etc. The infection caused by microorganisms that are not present in the patient at the time of admission to hospital but develop during the course of the stay in hospital are called hospital acquired infection or nosocomial infections. ${ }^{8}$

Bacteria are the major group of microorganisms that cause these infections, bacteria commonly associated with hospital infections include, klebsiellapneumuniae, Escherichia coli, Pseudomonas aeruginosa, serratiamarcescens staphylococcus epidermidis, proteus mirabilis, staphylococcus aureus etc, these bacteria are primarily transmitted through the direct contact with the body of healthcare personnel (hands and clothes), hospital equipment, devices and materials. ${ }^{9}$ This results in prolonged hospital stay, increase in the cost of treatment, immobilization of patients and even death of the patient in some extreme cases. ${ }^{10}$ However, not all colonization results in such infections and diseases. The infections at times could be mild resulting in no illness. The standard of hygiene in hospitals is determined by the workers, patient and the sterilization techniques applied. ${ }^{11}$ In achieving this, the medical personnel are the most important and their 
activities determine the extent of hygiene in the hospital environment. Hospital hygiene is a measure of cleanliness in the hospital and its environs in order to avoid the spread of infectious diseases and thus promote good health. ${ }^{12}$

The bacteria associated with hospital infection may be acquired indirectly through various vehicles or by direct contact. A variety of vehicles have been implicated in the spread of nosocomial infections. For example, most urinary tract infections (UTIS) are due to multiple drug resistant hospital pathogens associated with hospital devices of the urinary tracts such as E.coli. ${ }^{13}$ Occasionally, transmission may be through members of the hospital staff who are carrying pathogens on their hands and bodies or clothes. However passive carriage on the hand of medical personnel constitutes the major mode of transmission. ${ }^{14}$

The purpose of the study was to: Determine the rate of nosocomial infections among healthcare personnel in Otukpo General Hospital, isolate and identify the microorganisms responsible for nosocomial infections, relate the microorganisms identified to different categories of medical staff and to draw a comparison on the load of microbes among the three categories of the subjects.

\section{Materials and methods}

\section{Study design}

This was a Cross-sectional study involving the use of quantitative methods for data collection in Otukpo, Benue State.

\section{Study area}

Otukpo is one of the towns in Benue State, located in the North central geopolitical zone of Nigeria on latitude $7.51 \mathrm{~N}$ and longitude 8.53E. The state has a total land mass of 38,000 square kilometers and a population of about 330,712 comprising 169,398 males and 161,314 female. ${ }^{15}$ Otukpo General Hospital is a hospital with about 10 department and 2,000 medical staff. The main occupation of the people is civil service. There are however farmers, traders and few hunters. The climate is tropical with two main seasons from April to October covering the rainy season and from November to March covering the dry season.

\section{Study population}

This study was conducted among the health care professionals notably the nurses, doctors and the ward attendants in general hospital otukpo spanning from February to May, 2016.

\section{Specimen collection}

One hundred and fifty sterile swabs sticks (commercially obtained) were used for sample collection. For isolations of the hospital personnel, beds, bedpan, chairs etc swabs sticks impregnated with sterile saline solution were rubbed on the right hand fingers of the hospital personnel and the hospital fomites. Culture media used were MacConkey agar (Oxoid), Chocolate agar (Oxoid) and Nutrient ager (oxoid).

Samples were taken from fomites such as beds, bedpans, chairs etc and from hospital personnel before and after hand wash, and after washing with disinfectants $(80 \%$ alcohol, medicated soap etc) used swab sticks were dipped into peptone water before $24 \mathrm{hrs}$ of incubation at $37^{\circ} \mathrm{C}$. One (1) $\mathrm{ml}$ of the broth culture was inoculated onto prepared plate of mac Conkey agar, Nutrient agar and chocolate agar. The plates were incubated for 18 hours and the pure cultures of the isolates stored on nutrient agar slant at $4{ }^{\circ} \mathrm{C}$ until needed.

\section{Data analysis}

The data obtained was analyzed using descriptive statistics such as frequency distribution, percentages, and means and corresponding standard deviations; inferential statistics such as Chi-square test for testing the association between categorical variables and independent T-test for examining differences between groups means of categorical and continuous data variables. The statistical significance of all results was considered when the $p$ value is $<0.05 .^{16}$

\section{Ethical considerations}

Ethical approval was obtained from the State Ministry of Health Human Research Ethics Committee, Makurdi before proceeding with the study. Informed consent was sought from each respondent before questionnaires were administered and taking of samples for laboratory analysis. The privacy, dignity, and autonomy of the respondents were maintained accordingly throughout the conduct of the study.

\section{Identification of isolates}

Initial identification of bacteria isolated was based on their cultural and morphological characteristics. Further identification was by biochemical characteristics employing standard procedures.

\section{Results}

This study shows that microorganisms inhabit the hands of health care personnel and equipment and materials in use in hospitals. The load of microorganisms were highest among the fomites, followed by those that washed with soap and water only and the least was recorded among the health care professionals who washed their hands with disinfectants. Ninety nine bacterial isolates were recovered in this: forty (40) isolates from health care personnel who washed their hands with disinfectant, another forty (40) from those who washed their hands with soap and water only and nineteen (19) from the hospital fomites.

According to Table 1; S.aureus had the highest contamination rate followed by E. coli, Proteus mirabilis and finally Pseudomonas spp. In males, $50 \%$ of the isolated microorganisms were $S$. aureus whereas in females, $S$. aureus was $45 \%$.

According to Table 2; Pseudomona spp. had the least rate of occurrence and were isolated only from nurses. As show in Table $3, S$. aureus occurred most in VIPs ward $(66.7 \%)$, followed by emergency pediatrics' unit, female surgical ward and finally children's ward. Pseudomonas spp with the least percentage occurrence was isolated only from male surgical ward and female surgical ward. Table 4 shows that rate of $S$. aureus contamination was higher in females $(47.8 \%)$ than in males $(41.2 \%)$.

As in Table 5, P. mirabilis had the least percentage occurrence of $5.0 \%$ and it was isolated only from nurses who washed their hands with soap and water only. Eighteen (45.0\%) of the isolated organisms were $S$. aureus and it occurred more among nurses than among attendants and doctors. As presented in Table 6, S. arueus was highest in children's wards followed by the emergency pediatrics' unit, female surgical ward, VIPs ward and male surgical ward respectively. Proteus mirabilis with the least percentage occurrence was isolated only from children and male surgical ward.

Out of the 19 microorganisms isolated, 8(42.1\%) were $S$. aureus and these occurred most on the sink followed by beds and bedpans. As shown in the Table 7, bedspreads and chair had the same rate of percentage occurrence (25.0\%). Proteus mirabilis which is the 
least isolated organism was recovered from bedspread. As shown in Table 8, E.coli ranked highest in children's ward with a percentage occurrence of $66.7 \% \mathrm{~S}$. aureus occurred most among male medical ward followed by VIPs wards, children's ward, female medical ward and finally emergency pediatrics unit. Proteus mirabilis with the least rate of occurrence was isolated only from male medical ward.

Table I Frequency of bacteria isolated according to sex of medical personnel who washed their hands with disinfectants

\begin{tabular}{llll}
\hline Microorganism & Male (\%) & Female (\%) & Total (\%) \\
\hline S. aureus & $10(50)$ & $9(45.0)$ & $19(47.5)$ \\
E. coli & $3(15.0)$ & $5(25.0)$ & $8(20.0)$ \\
P. mirabilis & $2(10.0)$ & $3(15.0)$ & $5(12.0)$ \\
K. pneumoniae & $3(15.0)$ & - & $3(7.5)$ \\
S. epidermidis & - & $3(15.0)$ & $3(7.5)$ \\
Pseudomonas spp & $2(10.0)$ & - & $2(5.0)$ \\
Total & $20(100.0)$ & $20(100.0)$ & $40(100.0)$ \\
\hline
\end{tabular}

Table 2 Incidence of bacteria isolated from the hands of the medical personnel according to staff designation

\begin{tabular}{|c|c|c|c|c|}
\hline Microorganism & Nurses (\%) & Doctors (\%) & Attendants (\%) & Total (\%) \\
\hline S. aureus & $5(29.4)$ & $13(6 \mid .9)$ & $I(50.0)$ & 19(47.5) \\
\hline E. coli & $4(23.5)$ & $3(14.3)$ & I (50.0) & $8(20.0)$ \\
\hline P. mirabilis & $4(23.5)$ & $\mathrm{I}(4.8)$ & - & $5(12.0)$ \\
\hline K. pneumoniae & $2(11.8)$ & $\mathrm{I}(4.8)$ & - & $3(7.5)$ \\
\hline S. epidermidis & - & $3(14.3)$ & - & $3(7.5)$ \\
\hline Pseudomonas spp & $2(11.8)$ & - & - & $2(5.0)$ \\
\hline Total & $17(100.0)$ & $21(100.0)$ & $2(100.0)$ & $40(100.0)$ \\
\hline
\end{tabular}

Table 3 Occurrence of bacteria isolated from the hands of medical personnel according to ward

\begin{tabular}{|c|c|c|c|c|c|c|}
\hline Microorganism & Children (\%) & Emergency (\%) & Female surgical (\%) & Male surgical & VIP (\%) & Total (\%) \\
\hline S. aureus & $4(33.3)$ & $5(60.0)$ & $5(55.6)$ & $3(37.5)$ & $4(66.7)$ & $19(47.5)$ \\
\hline E. coli & $5(4 I .7)$ & & $\mathrm{I}(20.0)$ & I(II.I) & $\mathrm{I}(12.5)$ & $8(20.0)$ \\
\hline P. mirabilis & $2(16.7)$ & - & - & $5(37.5)$ & - & $5(12.0)$ \\
\hline K. Pneumoniae & - & - & - & $2(22.2)$ & $\mathrm{I}(\mathrm{I} 6.7)$ & $3(7.5)$ \\
\hline S. epidermis & $\mathrm{I}(8.3)$ & & $I(20.0)$ & - & $\mathrm{I}(16.7)$ & $3(7.5)$ \\
\hline Pseudomonas spp & - & - & $I(I I . I)$ & $\mathrm{I}(\mathrm{I} 2.5)$ & - & $2(5.0)$ \\
\hline Total & $12(100.0)$ & $5(100.0)$ & $8(100.0)$ & $8(100.0)$ & $6(100)$ & $40(100)$ \\
\hline
\end{tabular}

Table 4 Bacteria isolated from medical personnel who washed their hands with soap and water only

\begin{tabular}{llll}
\hline Microorganism & Male (\%) & Female (\%) & Total (\%) \\
\hline S.aureus & $7(4 I .2)$ & $\mathrm{II}(47.8)$ & $18(47.5)$ \\
E. coli & $4(23.5)$ & $4(17.4)$ & $8(20.0)$ \\
K. pneumoniae & $\mathrm{I}(5.9)$ & $4(17.4)$ & $5(12.0)$ \\
S. epidermidis & $2(11.8)$ & $2(87)$ & $4(10.0)$ \\
Pseudomonas spp & $3(17.6)$ & - & $3(7.5)$ \\
P. mirabilis & - & $2(87)$ & $2(5.0)$ \\
Total & $17(100.0)$ & $23(100.0)$ & $40(100.0)$ \\
\hline
\end{tabular}

Table 5 Frequency of bacteria isolated from the hands of the medical personnel according to staff designation

\begin{tabular}{|c|c|c|c|c|}
\hline Microorganism & Nurses (\%) & Doctors (\%) & Attendants (\%) & Total (\%) \\
\hline S. aureus & $10(52.6)$ & $7(36.8)$ & $\mathrm{I}(50.0)$ & $18(45.0)$ \\
\hline E. coli & $6(31.6)$ & $\mathrm{I}(5.3)$ & $I(5.0)$ & $8(20.0)$ \\
\hline K. pneumonia & $\mathrm{I}(5.3)$ & $4(21.1)$ & - & $5(12.0)$ \\
\hline S. epidermidis & $4(21.1)$ & - & & $4(10.0)$ \\
\hline Pseudomonas Spp & $3(15.8)$ & - & & $3(7.5)$ \\
\hline P. mirabilis & $2(10.5)$ & - & & $2(5.0)$ \\
\hline Total & $19(100.0)$ & $19(100.0)$ & $2(100.0)$ & $40(100.0)$ \\
\hline
\end{tabular}

Table 6 Occurrence of bacteria isolated from the hands of the medical personnel according to ward

\begin{tabular}{|c|c|c|c|c|c|c|}
\hline Microorganism & Children (\%) & Emergency (\%) & Female Surgical (\%) & Male Surgical (\%) & VIPS (\%) & Total (\%) \\
\hline S. aureus & $6(75.0)$ & $3(50.0)$ & $5(45.5)$ & $2(20.0)$ & $2(40.0)$ & $18(45.0)$ \\
\hline E. coli & - & $-2(33.3)$ & $3(27.3)$ & $2(20.0)$ & $I(20.0)$ & $8(20.0)$ \\
\hline K. pneumoniae & - & $\mathrm{I}(\mathrm{I} 6.7)$ & $3(27.3)$ & $I(10.0)$ & - & $5(12.0)$ \\
\hline S. epidermidis & $\mathrm{I}(12.5)$ & - & - & $I(10.0)$ & $2(40.0)$ & $4(10.0)$ \\
\hline Pseudomonas spp & & - & $-3(30.0)$ & - & $3(7.5)$ & \\
\hline P. mirabilis & $I(I 2.5)$ & & - & $I(I 0.0)$ & - & $2(5.0)$ \\
\hline Total & $8(100.0)$ & $6(100.0)$ & $\mathrm{II}(100.0)$ & $10(100.0)$ & $5(100.0)$ & $40(100.0)$ \\
\hline
\end{tabular}


Table 7 Frequency of bacteria isolated from the hospital fomites

\begin{tabular}{|c|c|c|c|c|c|c|c|}
\hline Microorganism & Bed (\%) & Bedpan (\%) & Bedspread (\%) & Chair (\%) & Cupboard (\%) & Sink (\%) & Total (\%) \\
\hline S. aureus & $3(75)$ & $2(50)$ & $\mathrm{I}(25)$ & $I(25)$ & - & $\mathrm{I}(100)$ & $8(42.1)$ \\
\hline E. coli & - & $\mathrm{I}(25)$ & $\mathrm{I}(25)$ & $\mathrm{I}(25)$ & $I(50)$ & & $4(21.1)$ \\
\hline K. pneumonia & - & $\mathrm{I}(25)$ & $\mathrm{I}(25)$ & $I(50)$ & & & $3(15.8)$ \\
\hline S. epidermidis & $\mathrm{I}(25)$ & - & $\mathrm{I}(25)$ & $I(25)$ & - & & $3(15.8)$ \\
\hline P. mirabilis & & - & $I(25 I)$ & & & - & $\mathrm{I}(5.8)$ \\
\hline Total & $4(100)$ & $4(100)$ & $4(100)$ & $4(100)$ & $2(100 \%)$ & $\mathrm{I}(\mathrm{I00})$ & $19(100)$ \\
\hline
\end{tabular}

Table 8 Occurrence of bacteria isolated from the hospital fomites according to ward

\begin{tabular}{|c|c|c|c|c|c|c|}
\hline Microorganism & Children & Emergency & Female surgical & Male surgical & VIPs & Total \\
\hline S. aureus & $\mathrm{I}(33.3 \%)$ & $\mathrm{I}(25.0 \%)$ & $\mathrm{I}(33.3 \%)$ & $4(57.1 \%)$ & $\mathrm{I}(50.0 \%)$ & $8(42.1 \%)$ \\
\hline E. coli & $2(66.7 \%)$ & - & $\mathrm{I}(33.3 \%)$ & $\mathrm{I}(\mathrm{I} 4.3 \%)$ & - & $4(21.1 \%)$ \\
\hline S. epidermidis & - & $2(50.0 \%)$ & - & - & $I(50.0 \%)$ & $3(15.8 \%)$ \\
\hline K. pneumonia & - & $\mathrm{I}(25.0 \%)$ & $\mathrm{I}(33.3 \%)$ & $\mathrm{I}(\mathrm{I} 4.3 \%)$ & - & $3(15.8 \%)$ \\
\hline P. mirabilis & - & - & - & $\mathrm{I}(\mathrm{I} 4.3 \%)$ & - & $\mathrm{I}(5.3 \%)$ \\
\hline Total & $3(100.0 \%)$ & $4(100.0 \%)$ & $3(100.0 \%)$ & $7(100.0 \%)$ & $2(100.0 \%)$ & $19(100.0 \%)$ \\
\hline
\end{tabular}

\section{Discussion}

Infections due to microorganisms are common all over the world as the causative agents are ubiquitous. The recognition of the role of health care personnel in the transmission particularly of hospital acquired infections led to attempts to reduce and prevent the incidence of such cross-infections. The healthcare professionals who washed with disinfectant recorded the lowest rate of contamination with the most and least isolated organisms being $S$. aureus (50\%) and Pseudomonas spp (10\%) respectively, all recorded among the male hospital workers. Sequel to Table 1, the S.aureus was frequently associated the doctors with $61.9 \%$ and the Pseudomonas spp $(11.8 \%)$, however recorded the least rate of occurrence and were isolated only from the nurses. The ward with the highest rate of contamination was the children's ward with a total of 12 organisms isolated. The female and male surgical wards recorded a total of 8 isolates each, which was followed by the VIPs ${ }^{6}$ and the emergency ward recorded the least rate of microbial contamination. ${ }^{5}$

The female healthcare workers recorded the highest rate of contaminants among those people that washed their hands with soap and water, and this was followed by the male counterpart. ${ }^{15}$ The frequently occurring organism was S. aureus (47.8\%) among the females and the least was Pseudomonas spp (17.6\%) found predominantly among the males. Proteus mirabilis had the least percentage occurrence of $5 \%$ and it was isolated only from the nurses who washed their hands with soap and water only. Forty five percent of the isolated organisms were $S$. aureus and it occurred more among the nurses than among the attendants and doctors. The female surgical ward had the highest rate of bacterial contamination; this was followed by the male surgical ward, children's ward and the emergency pediatrics ward. The least rate of contamination was recorded in VIPs ward. Staphylococcus aureus was highest in children's wards followed by the emergency pediatrics' unit, female surgical, VIPs ward and male surgical ward respectively. Proteus mirabilis with the least percentage occurrence was isolated only from children's and male surgical wards.

The beds, bedpans, bedspreads and chairs recorded the highest rate of bacterial contamination. The least occurrence organism was found on the sink. Out of the 19 microorganisms isolated, 8(42.1\%) were $S$. aureus and these occurred most on the sink followed by beds and bedpans. As shown in the table, bedspreads and chair had the same rate of percentage occurrence $(25.0 \%)$. Proteus mirabilis which is the least isolated organism was recovered from bedspread. Male surgical ward had the highest rate of contamination; this was followed by emergency ward. The female surgical and children's ward had the same rate of contamination. E.coli ranked highest in children's ward with a percentage occurrence of $66.7 \% \mathrm{~S}$. aureus occurred most among male medical ward followed by VIPs wards, children's ward, female medical ward and finally emergency pediatrics unit. Proteus mirabilis with the least rate of occurrence was isolated only from male medical ward. There was no significance difference in the organisms isolated between categories of healthcare workers who washed with disinfectant and those who washed with soap and water only when $\mathrm{p}<0.05$.

The result obtained from this study indicates that microorganisms inhabit the hands of health care personnel and equipment and materials in use in hospitals. Ostensibly the load of microorganisms were highest among the fomites, followed by those that washed with soap and water only and the least was recorded among the health care professionals who washed their hands with disinfectants. As reported by Hart, ${ }^{17}$ those microorganisms isolated were the major causes of hospital infections. This result agrees with various studies carried out in Europe and America that the high prevalence of staphylococcus aureus is probably related to the symbiotic association formed with an unimpaired immunity and intact anatomical structure of the human host. ${ }^{18}$ Proteus mirabilis frequently isolated among the males notably the elderly might be as a result of their diminished immunity and this result agrees with studies carried out in London. ${ }^{19}$

The high incidence of $E$. coli in males in contrast to females might be as a result of benign prostatic enlargement in men which is the most predisposing factor. This is so because of the frequency and methods in which catheters are introduced to ease urination. $E$. coli being a normal flora in the gastrointestinal tract may mistakenly be introduced into the patient when established protocol designed for sterility is not followed and this result agrees with study carried out in Edinburgh. ${ }^{20}$

Hospital acquired infections are a concern of health care providers. Numerous studies have shown that hospitalized patients are at increased risk of becoming colonized with organisms, which are unique to the hospital environment especially methicillinresistant staphylococcus aureus. ${ }^{21}$ The organisms are carried on the hands of health care personnel probably from colonized patients to newly admitted patients who then become colonized themselves; therefore adequate washing of hands with disinfectant is a major way of preventing and controlling nosocomial infections. ${ }^{22}$ 
The major types of organisms isolated from the hands of the health carepersonnel and the hospital fomites in this study were Staphylococcus aureus, Eschenchia coli, Klebsiellapneumoniae and Pseudomonas spp. The most frequently isolated was Staphylococcus aureus. ${ }^{23}$. It should be noted that some of these bacteria are part of the normal floral of the skin therefore their total elimination from the hands might be difficult. At present the only way of reducing the number and types of bacteria from the hands of health care personnel is by maintaining good hand washing and hand disinfection practices. ${ }^{24} \mathrm{~A}$ study by Stein showed that there was a greater level of compliance to hand washing policies by nurses than doctors. Among the reasons given for poor compliance by doctor are time constraints. There has also been a mistaken view among the health profession also that they do not need to wash their hands because they wear gloves. Wearing gloves has been report to only reduce the amount of contamination on the hands and does not eliminate it because fluids can still get through microscopic holes in the gloves. ${ }^{25}$

\section{Conclusion}

The major types of organisms isolated from the hands of the health care personnel and the hospital fomites in this study were Staphylococcus aureus, Eschenchia coli, Klebsiellapneumoniae and Pseudomonas spp. The most frequently isolated was Staphylococcus aureus. It should be noted that some of these bacteria are part of the normal floral of the skin therefore their total elimination from the hands might be difficult. To ensure proper hygiene, medical personnel should consider staff decontamination following a period of nursing of patients especially if they are to attend to unaffected patients shortly. As further advances occur in medical care and limited health care resources are spread across hospital and community needs, Innovations in infection control need to be evaluated for efficacy and cost effectiveness. With this understanding, it is possible that hospital infection can be controlled and largely prevented. The dictum of Florence Nightingale, made over a century ago, that the very first requirement in a hospital is that it should do the sick no harm; remains the goal!

\section{Recommendation}

a. Generally, hands play an important role in the transmission of infections. Consequently, frequent hand washing and hand disinfection have become one of the main methods of reducing or preventing this menace (CDC, 2014). The procedures of hand disinfection must however be done in correct manner without any reluctance on the part of the personnel which may render the antiseptic process ineffective.

b. Hospital Personnel should always wash their hands before performing invasive procedures, and before and after taking care of particularly susceptible patients. Also antimicrobial hand washing products should be used after routine hand washing in order to eliminate organisms which might still be present on the hands after washing with plain soap and water. Hand washing facilities should be conveniently located throughout the hospital.

c. Equipment and materials for clinical use should be assessed to determine the appropriate method, frequency and site of decontamination.

d. The crucial strategy in avoiding the spread of bacteria is strict hygiene management through hand disinfection and the use of chemical disinfectants for walls, floors; furniture etc. The spread of multiple resistant bacteria can be controlled by judicious use of antimicrobials and compliance with basic rules of hygiene. Nurses should be baited to comply with the rules of antisepsis at intensive care units. The paramedical staff should also be encouraged to wash up their hands with detergents and disinfectants. Aseptic procedures should be observed when dressing patients and instruments should be sterilized properly.

\section{Limitations}

This work is limited in the area of sample size. With supported funds larger sample size would have been evaluated.

\section{Acknowledgments}

None.

\section{Conflicts of interest}

Authors have declared that no competing interests exist.

\section{Authors' contributions}

This work was carried out in collaboration between all authors. Author ISE designed the study, performed the statistical analysis, wrote the protocol, and wrote the first draft of the manuscript and managed literature searches. Authors IBOB managed the analyses of the study and literature searches. All authors read and approved the final manuscript.

\section{Significance statement}

This study discovers that microorganisms inhabit the hands of health care personnel and equipment and materials use in hospitals. The heaps of microorganisms were most astounding among the fomites, trailed by those that washed with just soap and water and the slightest was recorded among the healthcare team members who washed their hands with disinfectants. The crucial strategy in avoiding the spread of bacteria is strict hygiene management through hand disinfection and the use of chemical disinfectants for walls, floors; furniture etc. The spread of multiple resistant bacteria can be controlled by judicious use of antimicrobials and compliance with basic rules of hygiene.

\section{References}

1. Wenzel RP. Prevention and control of Nosocomial infection. 3rd edn, Lippincott, Williams and Wilkins Baltimore, USA. 2007.

2. WHO. Prevention of Hospital-acquired infections. A Practical Guide. World Health Organization, Switzerland, 2002. p. 1-3.

3. AMA. Costs of health care-associated infections:9.8 billion annually in US. American Medical Association, USA. 2013.

4. WHO. Patient Safety. WHO guidelines on hand hygiene 3669 in health care. World Health Organization, Geneva, Switzerland, 2015:3670.

5. Iquo Bassey Otu-Bassey, Itodo Sunday Ewaoche, Blessing Felix Okon, et al. Microbial Contamination of House Hold Refrigerators in Calabar Metropolis-Nigeria. American Journal of Epidemiology and Infectious Disease. 2017;5(1):1-7.

6. Report 2005 Hospital Infection controls. Guidance on the control of infection in Hospitals. Department of Health and public Health Laboratory Services, London, UK.

7. www.medical-devices.gov.UK

8. Griffith CJ, Gebhart F. Cracking down on Nosocomial Infections. 2013;461:23.

9. Ayliffe GAJ, Fraise A, Mitchell K, et al. Control of Hospital Infection. 4th edn, Arnold, London, UK. 2000. 
10. Ayliffe GJ, Babb JR, Quoraishi AH. A test for hygiene hand disinfection. J Clin Pathol. 1978;31(10):923-928.

11. www.epic.tvu.ac.UK

12. Boschert S. More hand washing seen with automated reminder (Fewer nosocomial Infections). Internal Medicine News. 2002;35(22):6.

13. Brock TD, Brock KM, Smith DW. Biology of Microorganism. 2013. pp. 296.

14. Boschert S. Hand hygiene program saved hospital $\$ 12$ million over 3 years. (Reduction in Nosocomial Infections). $O B$ GYN News. 2012;37(27):34.

15. Cochran WG. Sampling Techniques, Wiley, New York, USA. 1963.

16. Denenberg H. Fixing the healthcare delivery system. One simple, effective long overdue solution. American journal of Medicine. 2015;5(1):1-7.

17. Gayness RP, Edwards JR, Jarvis WR, et al. Nosocomial Infection among neonates in high risk nurseries in the United State, National Nosocomial Surveillance system. Pediatrics. 2012;98(3 Pt 1):357-361.

18. Griffith CJ, Malik RBS, Cooper R, et al. Environmental surface cleanliness and the potential for contamination during handwashing. American Journal of Infection Control. 2013;31(2):93-96.
19. Hingst V, Judilzki I, Heeg P, et al. Evaluation of the efficacy of surgical hand disinfection following a reduced application time of 3 instead of 5 minutes. J Hosp infect. 2012;20(2):79-86.

20. Karabey S, Ay P, Derbentli S, et al. Hand washing frequencies in an intensive care unit. J Hosp Infect. 2012;50(1):36-41.

21. Ayliffe GAJ, Fraise A, Mitchell K, et al. Control of Hospital Infection. $4^{\text {th }}$ edn. Arnold, London Centers for Disease Control Hospital infection program. 2000.

22. Lilly HA, Lowbury EJL. Detergent compared with each other and antiseptics as skindegerming agents. $J$ clin pathol. 1979;82(1):497-500.

23. Lilly HA, Lowbury EJL. Transient skin flora:their removal by cleansing or disinfection in relation to their mode of deposition. $J$ clin pathol. 1978:31:919-922.

24. Lowbury EJL, Lilly HA, Bull JP. Disinfection of hands:Removal of transient organisms. Br Med J. 1964;2(5403):230-233.

25. Mehta S, Tsakris A, Castro D, et al. The effect of Disinfectants on perforated gloves. J Hosp Infect. 1991;18:191-200. 